\title{
How to test NISP instrument for EUCLID mission in laboratory
}

Costille, A.; Carle, Michael; Fabron, Christophe; Prieto, Eric; Beaumont, Florent; Jessen, Niels Christian; Jakobsen, Peter; Sørensen, Anton N.; Andersen, Michael I.; Grupp, Frank

Total number of authors:

14

Published in:

Space Telescopes and Instrumentation 2016: Optical, Infrared, and Millimeter Wave

Link to article, DOI:

$10.1117 / 12.2231630$

Publication date:

2016

Document Version

Publisher's PDF, also known as Version of record

Link back to DTU Orbit

Citation (APA):

Costille, A., Carle, M., Fabron, C., Prieto, E., Beaumont, F., Jessen, N. C., Jakobsen, P., Sørensen, A. N., Andersen, M. I., Grupp, F., Maciaszek, T., Ealet, A., Gillard, W., \& Clemens, J-C. (2016). How to test NISP instrument for EUCLID mission in laboratory. In H. A. MacEwen, G. G. Fazio, \& M. Lystrup (Eds.), Space Telescopes and Instrumentation 2016: Optical, Infrared, and Millimeter Wave (Vol. 9904). [99042U] SPIE International Society for Optical Engineering. Proceedings of SPIE - The International Society for Optical Engineering https://doi.org/10.1117/12.2231630

\section{General rights}

Copyright and moral rights for the publications made accessible in the public portal are retained by the authors and/or other copyright owners and it is a condition of accessing publications that users recognise and abide by the legal requirements associated with these rights.

- Users may download and print one copy of any publication from the public portal for the purpose of private study or research.

- You may not further distribute the material or use it for any profit-making activity or commercial gain

- You may freely distribute the URL identifying the publication in the public portal 


\title{
How to test NISP instrument for EUCLID mission in laboratory
}

\author{
A. Costille*a, Michael Carle ${ }^{\mathrm{a}}$, Christophe Fabron ${ }^{\mathrm{a}}$, Eric Prieto ${ }^{\mathrm{a}}$, Florent Beaumont ${ }^{\mathrm{a}}$, Niels-Christian \\ Jessen $^{b}$, Peter Jakobsen ${ }^{c}$, Anton Norup Sørensen ${ }^{c}$, Michael Ingemann Andersen ${ }^{c}$, Frank Grupp ${ }^{d}$, \\ Thierry maciaszek ${ }^{\mathrm{e}, \mathrm{a}}$, Anne Ealet ${ }^{\mathrm{f}}$, William Gillard ${ }^{\mathrm{f}}$, jean-claude clemens ${ }^{\mathrm{f}}$ \\ aAix Marseille Université CNRS, LAM (Laboratoire d'Astrophysique de Marseille) UMR 7326, \\ 13388, Marseille, France, ${ }^{\mathrm{b}}$ DTU Space, ${ }^{\mathrm{c}}$ Dark Cosmology Centre, Niels Bohr Institute, University \\ of Copenhagen, ${ }^{\mathrm{d}}$ Max-Planck-Institut für extraterrestrische Physik, Germany, ${ }^{\mathrm{e}} \mathrm{Ctr}$. National d'Études \\ Spatiales, France, ${ }^{\mathrm{f}} \mathrm{Ctr}$. de Physique des Particules de Marseille, France
}

\begin{abstract}
The ESA mission Euclid is designed to explore the dark side of the Universe. The NISP (Near Infrared SpectroPhotometer) is one of its two instruments operating in the near-IR spectral region (0.9-2 $\mu \mathrm{m})$, that will be fully integrated and tested at Laboratory d'Astrophysique de Marseille (LAM) under vacuum and thermal conditions. The test campaign will regroup functional tests, performance tests, calibration procedure validation and observations scenario test. One of the main objectives of the test campaign will be the measurement of the focus position of NISP with respect to the EUCLID object plane. To achieve these tests campaign, a global Ground Support Equipment (GSE) called the Verification Ground System (VGS) has to be developed. It will be a complex set of GSE integrated in ERIOS chamber made of: a telescope simulator to simulate the EUCLID telescope and to inject light into NISP, a thermal environment to be used for NISP thermal balance and verification, a sets of mechanical interfaces to align all the parts into ERIOS chamber, the NISP Electrical GSE (EGSE) to control the instrument during the test and a metrology system to measure the positions of the components during the test. We will present the preliminary design and concepts of the VGS and we will show the main difficulties we have to deal with: design of thermal environment at $80 \mathrm{~K}$ with $4 \mathrm{mK}$ stability, the development of a metrology system in vacuum, knowledge of the focus position within $150 \mu \mathrm{m}$ in cold, etc. The main objectives of the NISP test will be explained and how the VGS responds to the test requirement.
\end{abstract}

Keywords: test configuration, thermal environment, vacuum, cryogenics, GSE

\section{INTRODUCTION}

EUCLID mission ${ }^{1}$ has been selected by ESA in 2012 in the context of the Cosmic Vision program to understand the nature of the dark energy and the dark matter. The mission is designed to map the geometry of the dark Universe by investigating the distance-redshift relationship and the evolution of cosmic structures thanks to two scientific instruments: the Near Infrared Spectroscopic Photometer (NISP) ${ }^{2}$ and the Visible Instrument (VIS) ${ }^{3}$. The NISP instrument of Euclid is dedicated to measure the redshift of millions of galaxies and to analyze their spatial distribution in the Universe thanks to two instrumental modes: the photometry uses broadband filters to acquire image of the galaxies, the spectroscopic mode used grisms to obtain there spectra. The broadband filters and grisms are mounted on two rotating wheels allowing to switch easily from mode to another and to acquire data of the same field of view of space.

NISP instrument is made by a European consortium led by Centre National des Etudes Spatiales (CNES) that includes laboratory and industries mainly from France, Germany, Italy and Spain. The NISP instrument will be fully assembled and aligned at Laboratoire d'Astrophysique de Marseille (LAM) in France. The Flight Model (FM) has also to be tested under vacuum and thermal conditions respectively in order to qualify the instrument in its operating environment and to perform the final acceptance test before delivery to the payload. The test campaign will regroup functional tests of the detectors and wheels, performance tests of the instrument, calibration procedure validation and observations scenario test, all done at LAM during two test campaign done before and after the vibration test campaign. One of the main objectives of these test campaigns will be the measurement of the focus position of NISP with respect to the EUCLID object plane.

*anne.costille@lam.fr; phone+33491055978; fax; +33491621190, www.lam.fr 
To achieve these tests campaign, a global Ground Support Equipment (GSE) called the Verification Ground System (VGS) is in development at LAM to fulfill the need for the NISP FM test. The VGS is a complex set of GSE, developed by LAM and its partners that will be used into the ERIOS chamber, a thermal vacuum chamber designed to test space instrument. We present in this article the different main technical specifications of the VGS and the main objectives of the NISP test campaign. Then we will describe the different components of the VGS. Current design and concepts chosen for the NISP test will be shown, in particular the trade-off made for the design of the thermal and mechanical GSE and the foreseen configuration for the test into ERIOS.

\section{NISP TEST CAMPAIGN DESCRIPTION}

\subsection{NISP overview}

The NISP instrument is fully described in [2] and shown in Figure 1 but we propose hereafter a rough overview of the instrument that is made of 3 main assemblies:

- The NI-OMA (Opto-Mechanical Assembly), composed of the Mechanical Support Structure (NI-SA) and its thermal control (NI-TC), the Optical elements (NI-OA), the Filter Wheel Assembly (NI-FWA), the Grism Wheel Assembly (NI-GWA), the Calibration Unit (NI-CU). The NI-OMA structure supports the Optical elements, the calibration unit, the Filter and Grism Wheel Units and the detection system. It provides the thermo-mechanical interface towards the Euclid PayLoad Module (PLM).

- The Focal Plane Array (NI-FPA) and the Sensor Chip System (NI-SCS) compose the Detector System Assembly (NI-DS). The NI-DS comprises the 16 H2RG detectors and associated 16 ASICS (Sidecars), passively cooled at operating temperature $(<100 \mathrm{~K}$ for the detectors; $140 \mathrm{~K}$ for the ASICS Sidecar). Thermal stabilization of the detector is "naturally" obtained thanks to the very good thermal stability provided by the Euclid PLM at the NISP interfaces

- The Warm Electronics Assembly (NI-WE), composed of the Instrument Data Processing Unit and Control Unit (NI-DPU/DCU), and the Instrument Control Unit (NI-ICU). The NI-ICU is managing the commanding and the control of the instrument. It is interfaced with the satellite via a 1553 bus. The NI-DPU/DCU controls the Sensor Chip System and basic image processing such as co-adding (DCU function) and the science onboard data processing, the compression and transfer of scientific data to the S/C Mass Memory using Spacewire links (DPU function). The NI-DPU/DCU functions are regrouped in a single mechanical box for controlling eight detectors. There are two NI-DPU/DCU boxes.

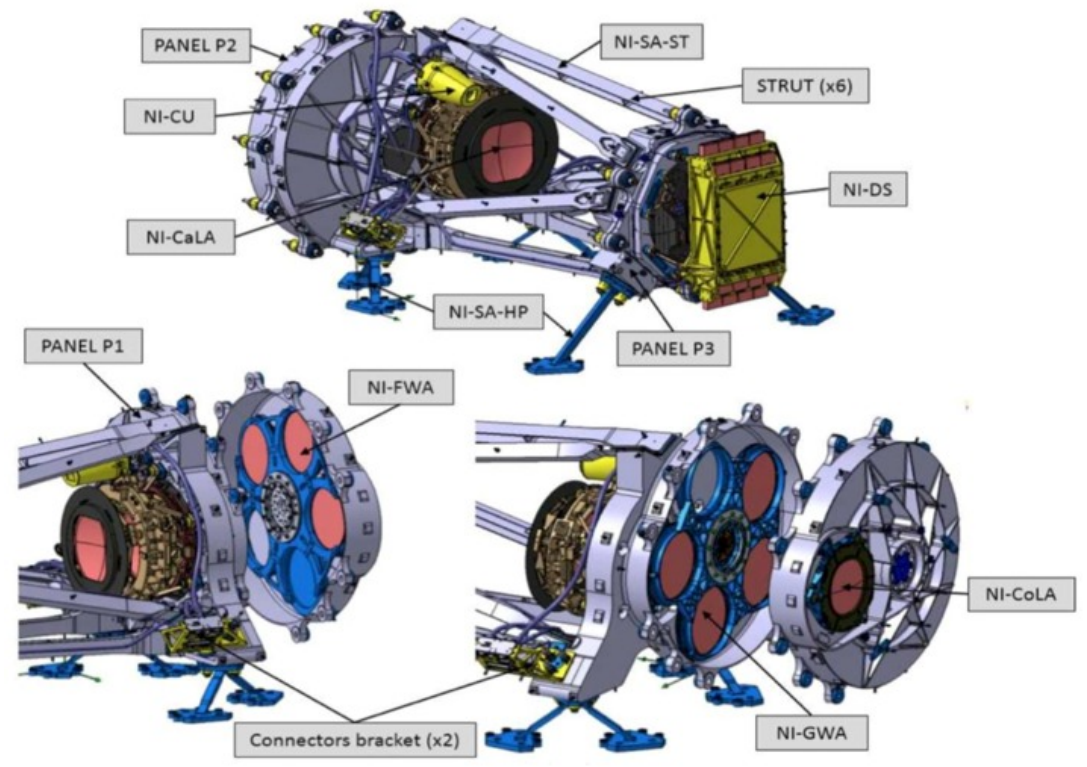

Figure 1. NISP instrument description 


\subsection{NISP test campaign goal}

NISP instrument will be fully integrated and test at LAM. In particular, two thermal performance tests of NISP FM instrument are foreseen to validate the instrument before and after the vibration of the FM. The test campaign is done in vacuum and cold and shall:

- Verify the performance requirement compliance of the NISP instrument. In particular a thermal balance will be performed to validate NISP thermal behavior and its thermal stability. The performance compliance in operational environment will be tested: detector noise (read-out and dark) at the coldest interface range, Point Spread Function (PSF) acquisition to measure encircled energy of NISP, full width half maximum of the images and image quality, limited plate scale measurement at the center and the corner of the Field of View (FoV), rough estimation of the stray-light sensitivity and check of the absence of ghosts;

- Provide the object plane position of NISP with respect to NISP reference frame at Operational Temperature (OT);

- Verify the functionality of NISP: observation scenario will be tested and validated including wheel, detector acquisition, data reduction and communication with spacecraft sequences;

- Provide information and strategy for the calibration of the instrument. The calibration scenario foreseen will be tested, data from ground calibration will be acquired and the in-flight calibration procedure will be verified through the acquisition of PSFs used for modeling of NISP PSF. The spectral dispersion law of the grisms will be measured.

The NISP test campaign will take place at LAM in ERIOS chamber, which is described in subsection 3.1. To achieve the goal of the test, a global Ground Support Equipment (GSE) called the Verification Ground System (VGS) is in development. This VGS surrounds EUCLID NISP in the ERIOS vacuum chamber and will permit to verify the performance and thermal environment requirements of the NISP and will provide a mean to test the instrument before the launch. This test campaign and the development of the VGS represent a great challenge as it will allow performing the test of NISP instrument performance on ground.

\subsection{VGS technical specifications}

The VGS has to answer to technical specifications provided by NISP Assembly Integration Verification (AIV) team. The main specifications are:

- To provide a thermal environment compatible with the flight environment of NISP: interface of the NISP Sidecar Support Structure (SSS) should be between 120K and 135K, interface of the NISP Cold Support Structure (CSS) between $80 \mathrm{~K}$ and $95 \mathrm{~K}$ and the NISP feet interface should be at $120 \mathrm{~K}$ to $135 \mathrm{~K}$. In addition, the VGS shall provide a radiative thermal interface to select temperature in a range between $80 \mathrm{~K}$ to $140 \mathrm{~K}$ with a stability of $+/-2 \mathrm{~K}$ during $1500 \mathrm{~s}$. Gradient of the conductive interface should be lower than $+/-1 \mathrm{~K}$ on the SSS, $+/-0.5 \mathrm{~K}$ for the CSS and +/-5K on NISP feet. Finally, NISP should be cooled down from Room Temperature (RT) to OT assuming $10 \mathrm{~K} / \mathrm{h}$ max at conductive interfaces. The CSS interface shall be kept at least $10 \mathrm{~K}$ warmer than the SSS and NISP first panel P1. The CSS shall be cooled down after the other parts to OT.

- To provide a simulation of EUCLID telescope to test NISP optical performance. The telescope should illuminate the NISP pupil with at least a F/20 beam and should provide object in the full NISP FoV covering +/$0.5^{\circ}$ of the sky. The source from the telescope should provide selectable monochromatic wavelength in the band 0.4 to $2.1 \mu \mathrm{m}$, multi-lines spectrum from 0.9 to $2 \mu \mathrm{m}$ and continuum spectrum from 0.9 to $2.02 \mu \mathrm{m}$. The flux of the source should be adjustable to test a large range of flux of NISP. The WaveFront Error (WFE) quality should be better than $30 \mathrm{~nm}$ rms over the FoV. The telescope should not introduce unexpected stray light to NISP FoV

- To provide a way to measure the reference coordinate system defined by laser tracker reflectors put on NISP instrument and the telescope simulator to allow an accurate metrology through the vacuum chamber. 


\section{DETAILED DESCRIPTON OF THE VGS}

\subsection{Overall test configuration}

To answer to the technical specifications presented in subsection 2.3, LAM and its partners have decided to develop a global GSE called the VGS made of:

- The NI-VTS, NISP Verification Telescope System: it is a telescope simulator of EUCLID telescope that injects light into NISP for optical and performance tests. The NI-VTS is developed by a Danish consortium between DTU space and Dark Cosmology Centre;

- The NI-TMVS, NISP Thermal and Mechanical Verification System: it is the thermal and mechanical environment to be used for NISP thermal balance and verification and all the thermal and mechanical interfaces needed for the test and to stabilize NISP in temperature. This part of the VGS is developed by LAM;

- The NI-MVS, NISP Metrology Verification System: it is a set of metrology tools used to measure the positions and orientations of the components during the test in the vacuum chamber. It is developed by LAM and a detailed presentation of the concept can be found in [4];

- The NI-WEVS, NISP Warm Electronic Verification System: it is the NISP Electrical GSE (EGSE) developed to control the instrument during the test on ground. This set of GSE is provided by an Italian consortium. More detailed can be found in [5] and in [6].

All the parts of the VGS will be installed into ERIOS chamber and a specific test configuration is under development. Figure 1 presents a view of the VGS for NISP test. NISP is installed on the center of ERIOS chamber, the NI-VTS is set in front of NISP to inject light into it, the NI-MVS is installed outside ERIOS on the entrance door to measure position through a window and to keep these parts warm. The TMVS corresponds to all the others parts shown in Figure 2.

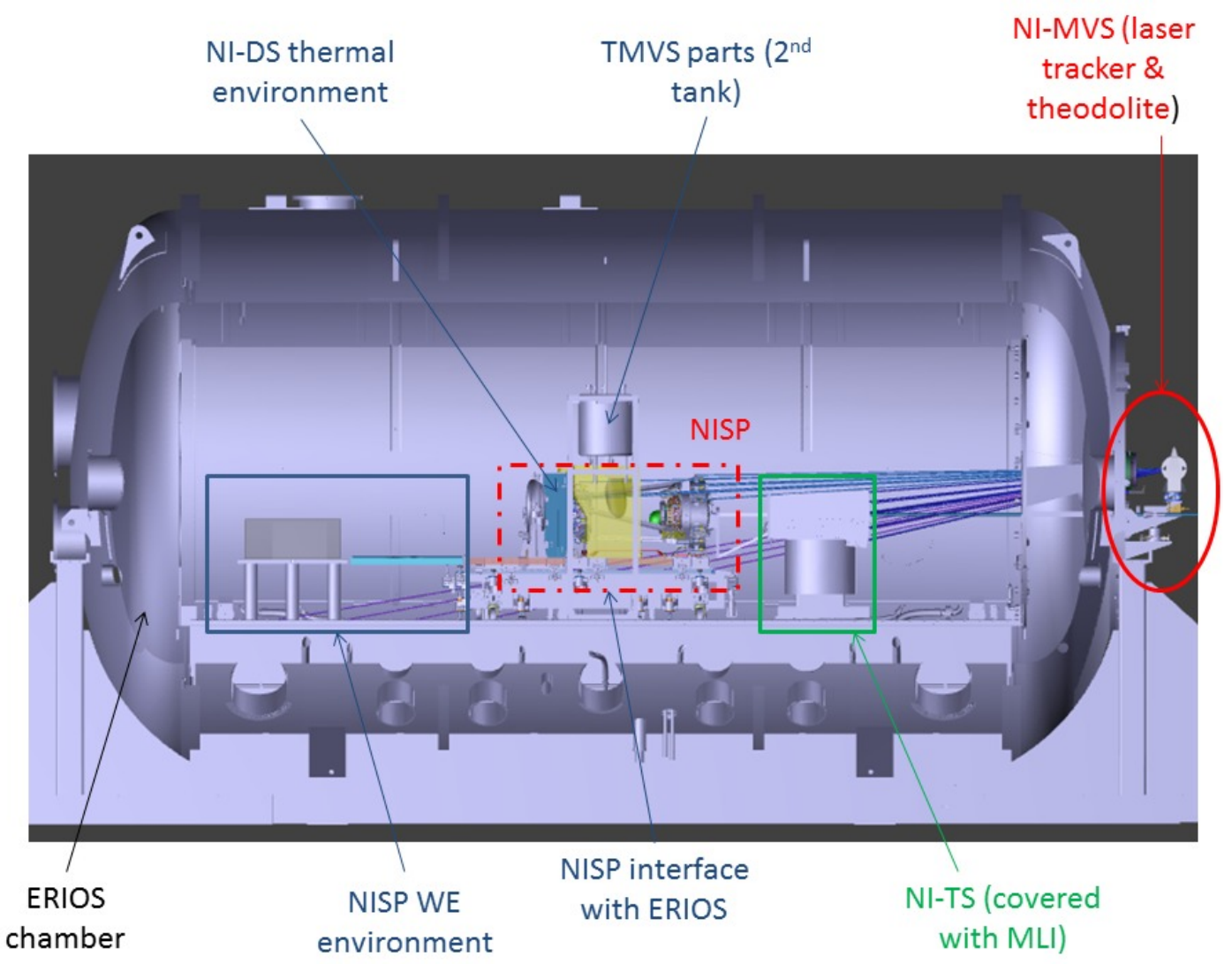




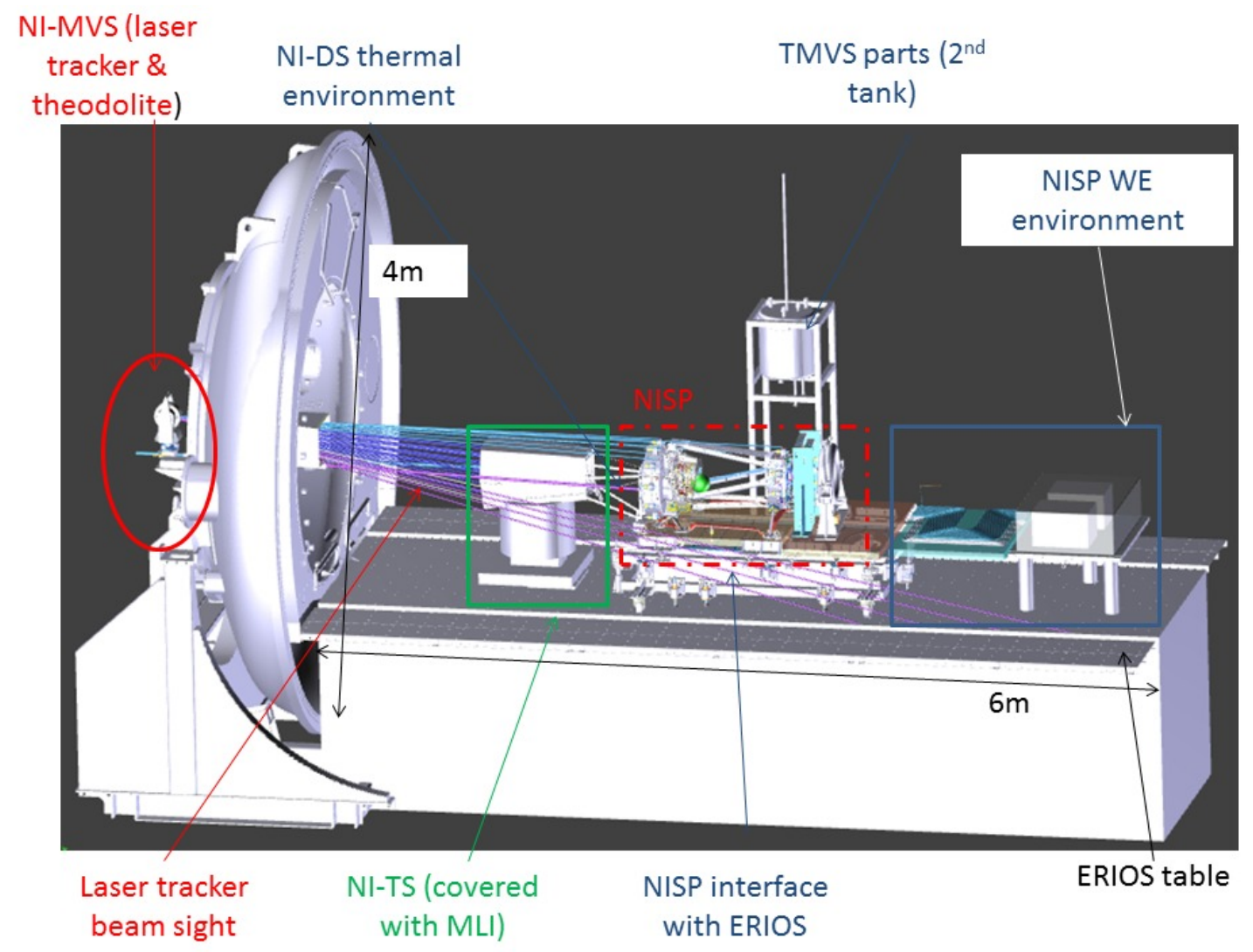

Figure 2. View of the VGS into ERIOS chamber. The different parts are shown.

We propose now to describe in detailed the different elements of the VGS to provide an overview of the complexity of this GSE.

\subsection{Erios chamber}

ERIOS is a large (the size of the envelope is 4 meters in diameter by 6 meters long) vacuum space simulation chamber owned by LAM for calibration, adjustment and integration for space optics which combines vacuum and temperature close to $-200^{\circ} \mathrm{C}$ and very high stability. To ensure stability, and hence accurate measurements, the instrument's supporting table is connected to a 100tone concrete block under the floor and rests on pillars via spring boxes.

ERIOS is equipped with fixed liquid nitrogen shrouds covering the entire inner part, the working volume (above the optical table) at cold temperature is $45 \mathrm{~m} 3$.

The pumping system is composed of one dry primary pump $\left(650 \mathrm{~m}^{3} / \mathrm{h}\right)$ and a root which allows reaching a pressure down to $5.10^{-2} \mathrm{mbar}$ in 4 hours, and the high vacuum $\left(\sim 10^{-6} \mathrm{mbar}\right)$ is reach using the two cryogenics pumps $(100001 / \mathrm{s})$. A liquid nitrogen cryopanel could be used for intermediate pumping and as cold trap to avoid any risk of molecular contamination of the instrument tested inside.

The main door of the chamber is equipped with a 2 meters diameter rotating flange which allow to position optical windows according to the desired optical sights inside the chamber.

The NISP STM model was the first space instrument to be tested in ERIOS. 


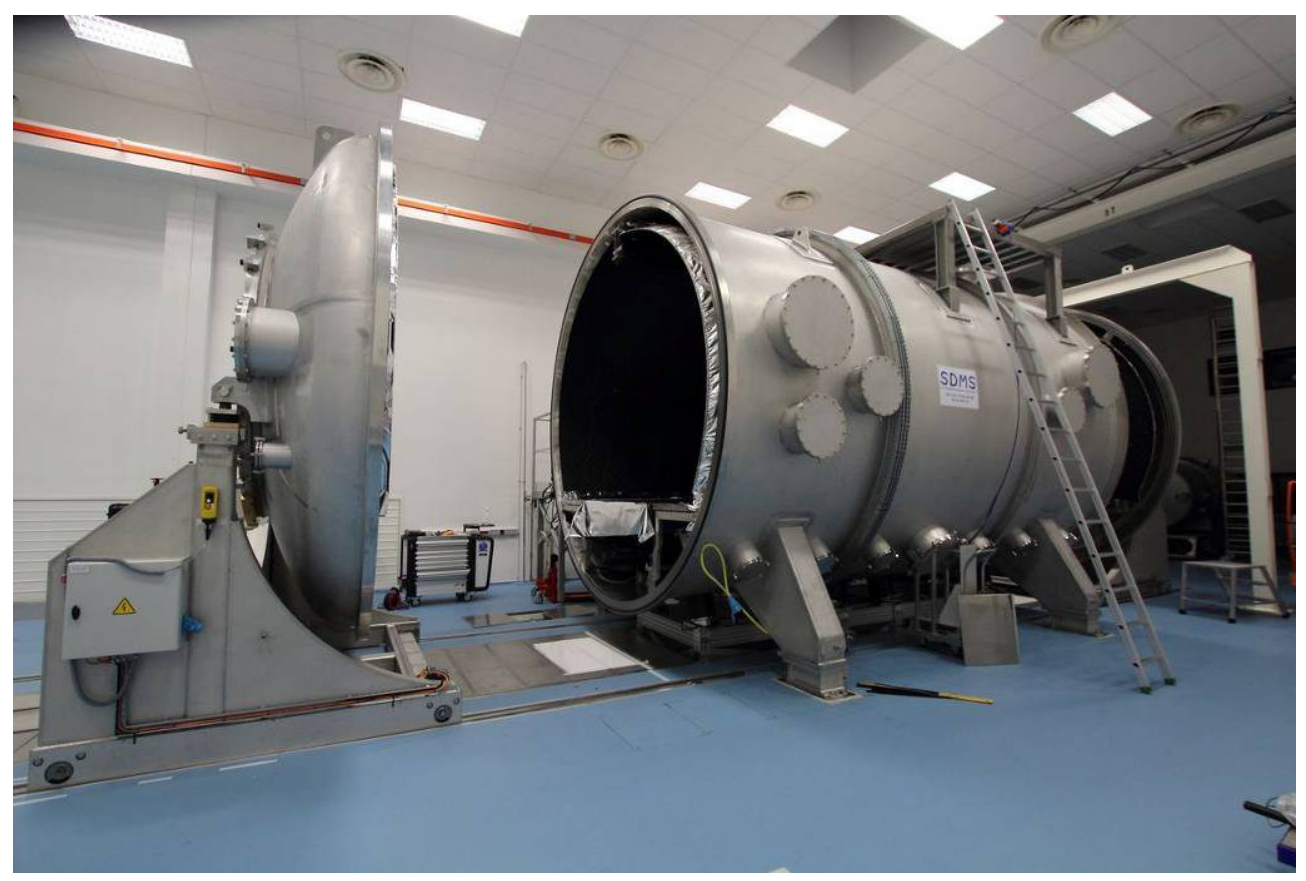

Figure 3: ERIOS facility

\subsection{The Optical Ground Support Equipment (OGSE): telescope simulator}

The concept of the OGSE developed for NISP is to simulate the F/20 EUCLID telescope beam thanks to an elliptical mirror positioned on stages that allow the telescope to move in five degrees of freedom to illuminate the entire NISP FoV. The OGSE is made of two main parts: the NISP Telescope Simulator (NI-TS) itself that mimics EUCLID telescope, and a Virtual Focal Plane (VFP) that is used to simulate the EUCLID object plane i.e where the focal plane will be in absence of NISP optics. The two parts are shown in Figure 4. The VFP is only used to validate the NI-TS functionality at cold during the acceptance of the VGS and will be not used during the test with NISP. The main goal of the NI-TS is to provide a stable focal distance to NISP to allow the measurement of the position of NISP object plane and on which we can rely on during this measurement. To achieve this goal, the NI-TS should be designed with a stable thermal environment done by the thermal flexures between the telescope itself and the motor assembly. This is one of the main challenges for the NI-TS design as the different parts of the NI-TS operate at different temperature and in a warmer environment than NISP.

The NI-TS is made of two units: the Active Telescope Support unit (ATS) that moves the second unit, the Telescope Simulator (TS), around to illuminate the full NISP FoV. The ATS is an assembly of 5 motors providing 5 degrees of freedom. It is made of 3 linear and 2 rotational high precision stages with remote controlled motor and absolute encoder to provide position of the unit. These stages are vacuum compatible and with a large moving range to commit with NISP need and in particular to allow a scanning of the focus to find NISP best focus. The ATS is directly in interface with ERIOS bench thanks to 3 shims under the last translation stage baseplate. The TS is made of an off-axis elliptical mirror assembled to a baseplate thanks to optical contact. On the same baseplate, a pinhole is set at the entrance focus to provide an interface for the source. It is a single surface optical design which provides an unobstructed system. The design of the TS is to provide a first focal point at $500 \mathrm{~mm}$ while the distance from the mirror vertex to the second focal point should be $3000 \mathrm{~mm}$. The whole TS assembly is made of Silica to ensure a good stability of the focal distance of the telescope and will operate at $165 \mathrm{~K}$ to limit impact of the temperature on the stability of the focal distance (the CTE of the silica being null at this temperature). The telescope is illuminated through a $2 \mu \mathrm{m}$ diameter pinhole placed in the primary focus and should provide uniform illumination of the $160 \mathrm{~mm}$ diameter aperture. The focus of the telescope should be known with a precision better than $20 \mu \mathrm{m}$ at operational temperature meaning that the distance from the pinhole to the mirror should be stable within $0.5 \mu \mathrm{m}$ and that an accurate measurement of the focal distance should be done at ambient temperature thanks to Coordinate Measurement Machine (CMM) with high precision and then at cold thanks to the MVS, described in subsection 3.5. The WFE should be lower than $30 \mathrm{~nm}$ rms over the $160 \mathrm{~mm}$ diameter aperture to 
ensure a good image quality on NISP during the performance test. A view of the telescope design is shown in Figure 4. In order to minimize stray light during NISP test, the telescope will be surrounded by a baffle made of several vanes.
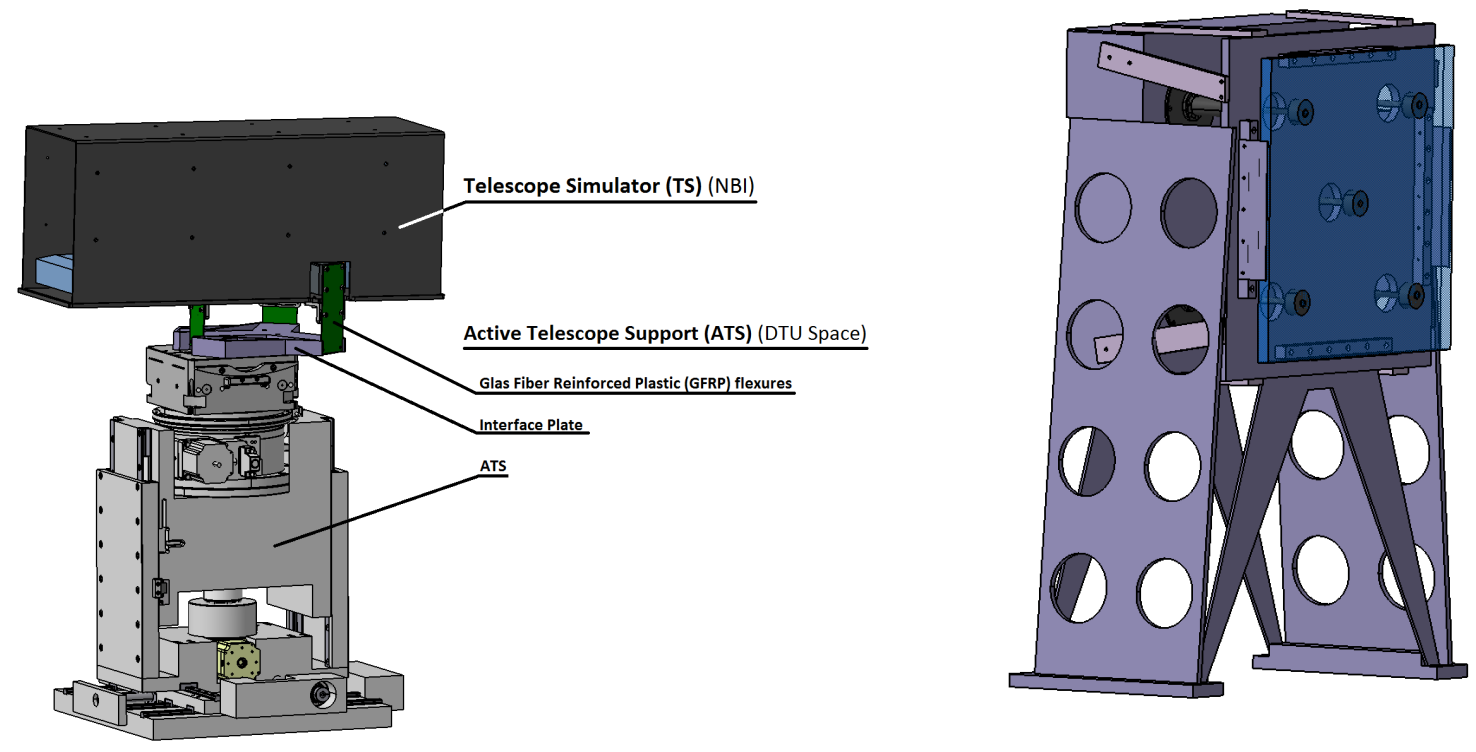

Figure 4. The two main parts of the OGSE. Left: the NI-TS without MLI showing the motor assembly and the telescope in its baffle, right: the VFP, the 5 focal unit are visible.
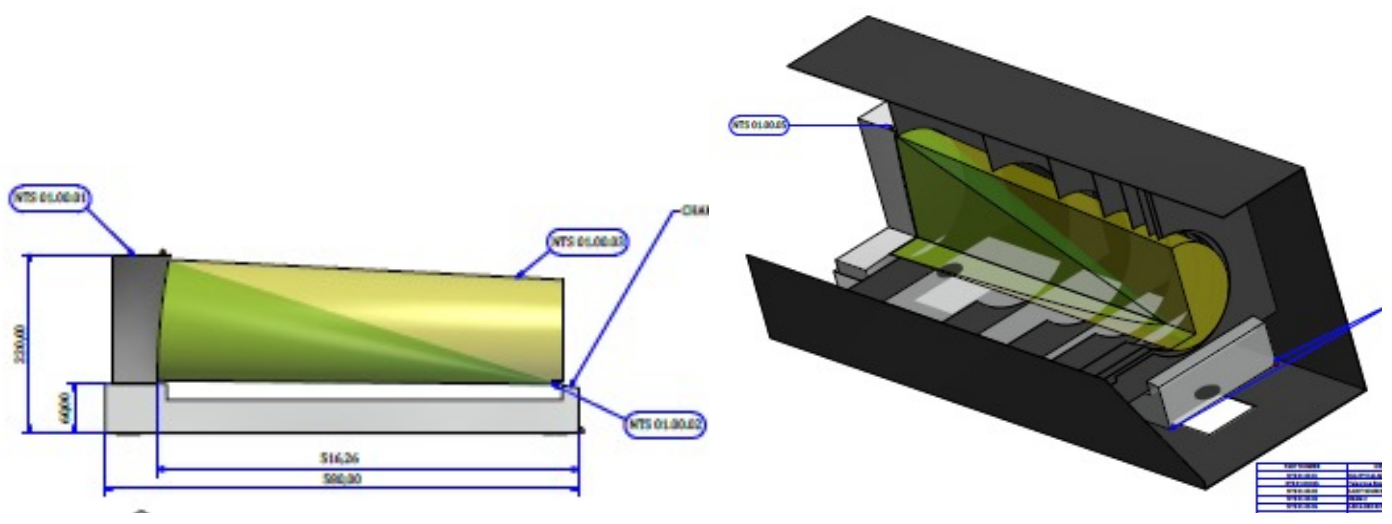

Figure 5. Left: view of the TS design, Right: view of the TS baffle design.

At the entrance of the TS, a fiber is connected to the illumination system used for the NI-TS. It is made of a monochromator that is fed from a continuum laser source. The output from the monochromator is delivered to a cryogenic fiber through a pair of vacuum windows in interface with ERIOS chamber. A cooled Neutral Density (ND) filter suppresses the thermal background. The flux can be adjusted in large steps via ND filters mounted in a filter wheel also serving as order-sorter for the grating based monochromator, The flux can be fine-tuned using a variable rotating ND filter. A spectral lamp and an etalon are available for wavelength calibration and can be selected using linear motions. The whole TS assembly will be installed into ERIOS vacuum chamber where liquid nitrogen cooled shrouds will be present. The ATS will be covered by MLI blanket to be kept warm and to avoid being cooled down by the cooling shrouds. The telescope itself will be kept at 165K. A thermal isolation between the ATS and TS will be present thanks to flexures made with a low conductivity material.

The VFP is used for the acceptance test of the NI-TS only and will not be present during NISP performance test. It is situated at the focal place of NISP in absence of NISP ie at NISP object plane and it should be coincident within $20 \mu \mathrm{m}$. The VFP is used to verify the position of the focal point of the NI-TS: a screen is observed through a simple optical system which reimages the focus screen onto a detector. A fine ground glass plate is preferred as it can be measured 
mechanically with high precision. The focus of the detector relative to the focus screen is monitored through the observation of the fiducial points on the screen. Figure 6 presents the principle of the focal plane unit. Five similar units are placed at 5 representative points of NISP FoV to verify the focal point in different point of the FoV and to ensure the stability and the good behaviour of the NI-TS assembly.

The NI-TS will be provided with a Telescope Control Computer (TCC) to control the motors position, the light illumination and intensity and to read data from the VFP.

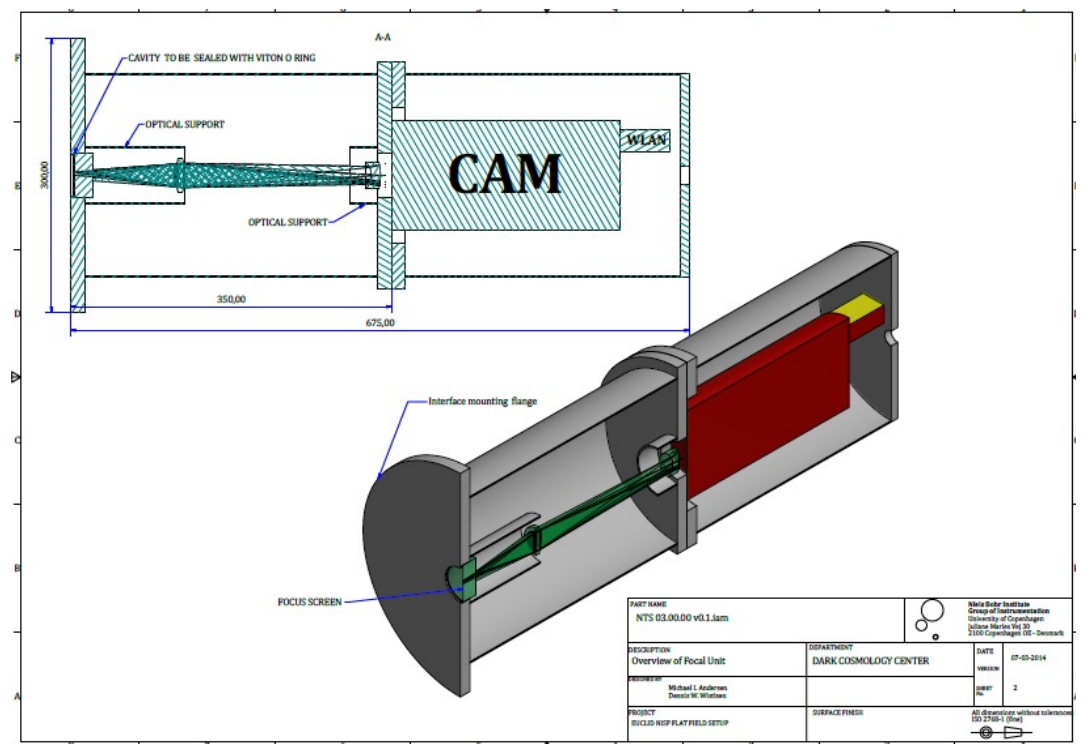

Figure 6. Focal plane unit.

\subsection{Thermal and mechanical ground support equipment}

The Thermal and Mechanical Ground Support Equipment also called TMVS provide thermal and mechanical interfaces for NISP. It should simulate the PLM thermal environment. The radiative thermal environment consists of ERIOS liquid nitrogen shrouds that covered NISP and VGS configuration. To avoid 300K background coming from the optical windows (for laser tracker and theodolite) a cold baffle is fixed between LN2 shrouds and the windows. Furthermore, a shutter is placed between this baffle and the windows. The shutter is activated from outside by a ferrofluid feed through which permits to close the shutter between two optical measurements.

The TMVS has also to provide conductive interfaces for NIOMADA, Warm Electronic and for the intermediate harness as described in Table 1.

\begin{tabular}{|c|c|c|c|c|c|}
\hline Case & & NI-CSS & NI-SSS & Feet & Comments \\
\hline \multirow{4}{*}{ cOT1 } & Temperature & $80 \mathrm{~K}+/-2 \mathrm{~K}$ & $120 \mathrm{~K}+/-2 \mathrm{~K}$ & $\begin{array}{l}120 \mathrm{~K}+7 /- \\
2 \mathrm{~K}\end{array}$ & \multirow{4}{*}{$\begin{array}{l}\text { The case is the } \\
\text { detector related } \\
\text { performance test } \\
\text { case }\end{array}$} \\
\hline & Stability & $\begin{array}{l}4 \mathrm{mK} / 1210 \mathrm{~s} \\
0.8 \mathrm{~K} / \mathrm{month}\end{array}$ & $\begin{array}{l}0.2 \mathrm{~K} / 1210 \mathrm{~s} \\
0.8 \mathrm{~K} / \mathrm{month}\end{array}$ & $\begin{array}{l}0.8 \mathrm{~K} / 1210 \mathrm{~s} \\
0.8 \mathrm{~K} / \mathrm{month}\end{array}$ & \\
\hline & Gradient & $+/-0.5 \mathrm{~K}$ & $+/-1 \mathrm{~K}$ & $+/-5 \mathrm{~K}$ & \\
\hline & Heat load & $2.3 \mathrm{~W}$ & $4 \mathrm{~W}$ & oW & \\
\hline \multirow{4}{*}{ cOT2 } & Temperature & $90 \mathrm{~K}+/-2 \mathrm{~K}$ & $130 \mathrm{~K}+/-2 \mathrm{~K}$ & $130 \mathrm{~K}+/-7 \mathrm{~K}$ & \multirow{4}{*}{$\begin{array}{c}\text { This case is the } \\
\text { optical } \\
\text { performance } \\
\text { stability and } \\
\text { Thermal balance }\end{array}$} \\
\hline & Stability & $\begin{array}{l}20 \mathrm{mK} / 1210 \mathrm{~s} \\
0.8 \mathrm{~K} / \mathrm{month}\end{array}$ & $\begin{array}{l}0.2 \mathrm{~K} / 1210 \mathrm{~s} \\
0.8 \mathrm{~K} / \mathrm{month}\end{array}$ & $\begin{array}{l}0.8 \mathrm{~K} / 1210 \mathrm{~s} \\
0.8 \mathrm{~K} / \mathrm{month}\end{array}$ & \\
\hline & Gradient & $+/-0.5 \mathrm{~K}$ & $+/-1 \mathrm{~K}$ & $+/-5 \mathrm{~K}$ & \\
\hline & Heat load & $2.3 \mathrm{~W}$ & $4 \mathrm{~W}$ & $0 \mathrm{~W}$ & \\
\hline cOT3 & Temperature & $95 \mathrm{~K}+/-2 \mathrm{~K}$ & $135 \mathrm{~K}+/-2 \mathrm{~K}$ & $135 \mathrm{~K}+/-7 \mathrm{~K}$ & This case is the \\
\hline
\end{tabular}




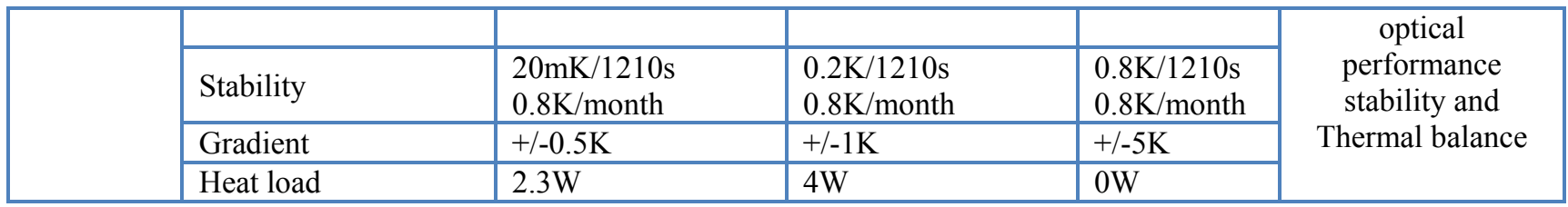

Table 1 Thermal Test case for NIOMADA

Table 1 presents the different thermal cases for NIOMADA. To meet these requirements, the TMVS is composed of four different parts:

- The cold plate in aluminium, linked to a liquid nitrogen tank placed under these table. This part of the thermal environment has been used for the NISP STM Thermal Balance Test. In the STM configuration, it was used as mechanical and thermal interface for NIOMA, NI-CSS thermal interfaces and NI-SSS thermal interfaces. The temperature of the plate is $90 \mathrm{~K}$ and the STM tests have shown a stability of $2.3 \mathrm{mK}$ over 1000 s (NISP exposure time) which was well within the stability required.

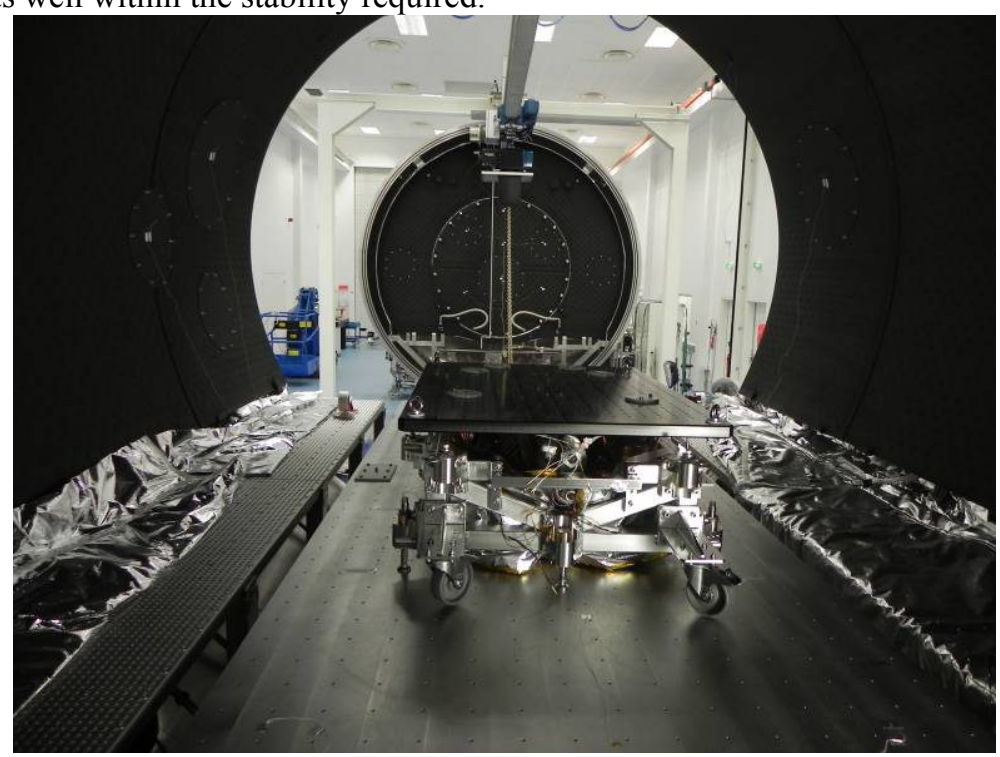

Figure 7: Cold plate in ERIOS before NISP STM Test campain

- The NISP Thermal Frame (NI-TF), a frame in Invar, is the mechanical interface with NISP feet. The frame is installed on the cold plate by an isostatic mount and is thermally linked to the plate by cold braids. To control the temperature (between $120 \mathrm{~K}$ and $135 \mathrm{~K}$ ) the frame is equipped with heaters uniformly distributed on the bottom side.

- The NI-CSS thermal interface is composed of four copper braids directly linked to an annular liquid nitrogen tank in order to ensure a cold case at $80 \mathrm{~K}$. Warmer temperatures and stability are ensured by heaters at the end of the braids controlled by a Lakeshore PID controller. The LN2 tank is supported by the aluminium cold plate.

- The NI-SSS is composed of brackets supported by the cold plate but insulated from it and controlled in temperature. Four thermal braids are linked between brackets and NI-SSS. The NI-SSS thermal interface shall also provide connector bracket at $130 \mathrm{~K}$ for SCE connectors. 

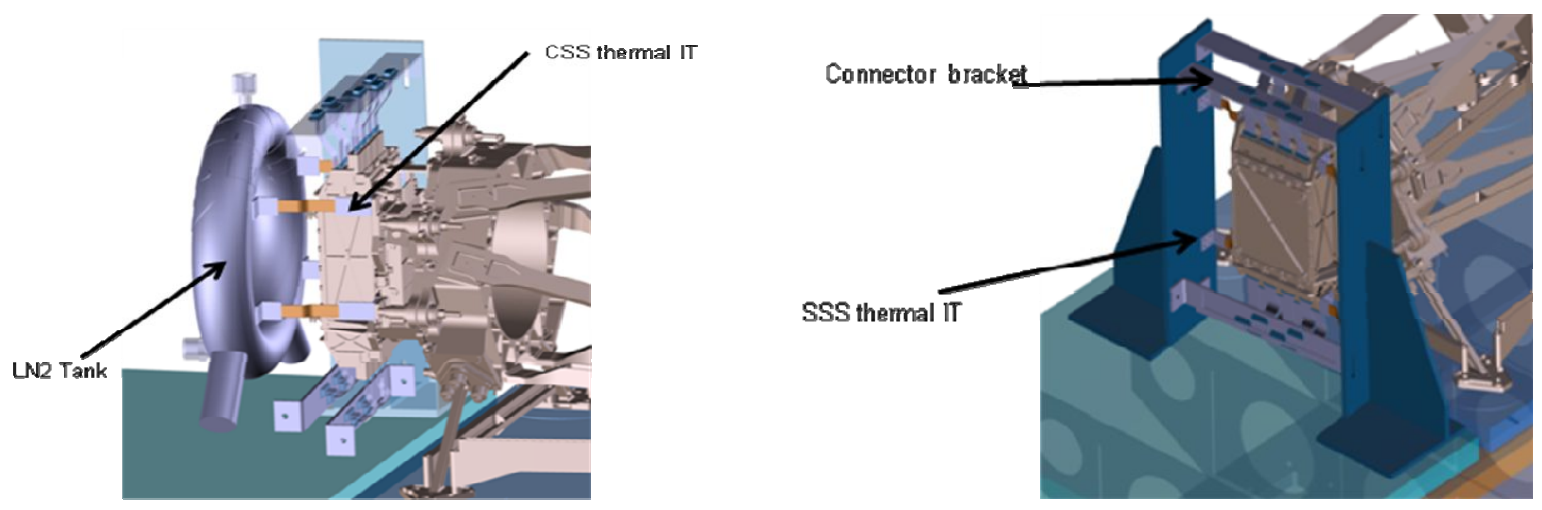

Figure 8: NI-CSS (left) and NI-SSS (right)Thermal interfaces

The liquid nitrogen loop to fill the tanks is shown Figure 9. A supply tank is positioned in the upper part of ERIOS. This tank permits to have autonomy of 30 hours between two fillings. It also permits to manage the cooling down phase in order to keep detectors warmer than the rest of the instrument.

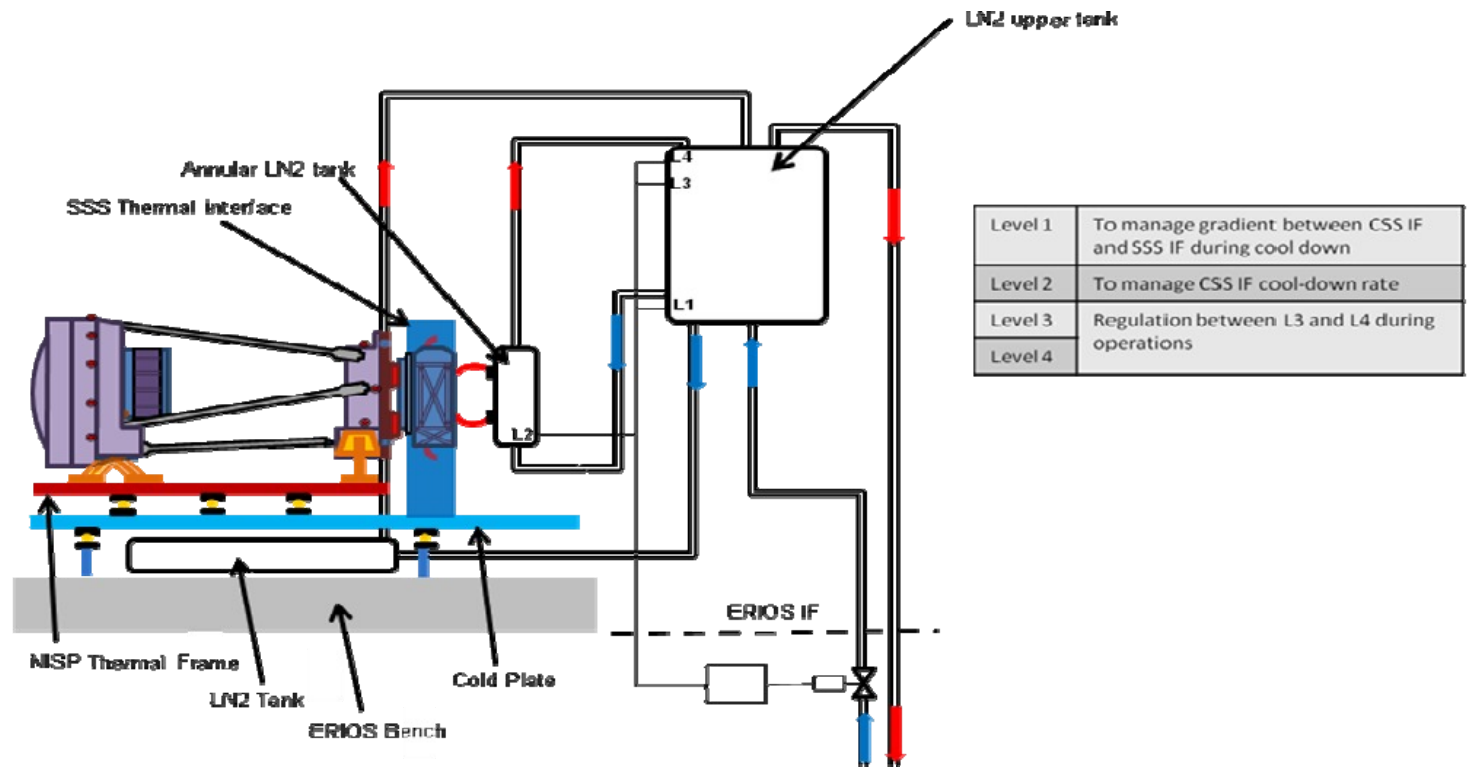

Figure 9: TGSE LN2 loop

In addition to NIOMADA, the TMVS has to provide conductive thermal interfaces for the harness and the warm electronic. The harness thermal unit is a stainless steel plate, where the NISP intermediate harness is fixed, with a temperature gradient between operational temperature $(130 \mathrm{~K})$ to room temperature. The cold part of the unit is linked by thermal braids to the NIOMADA cold plate and the warm part is regulated by heaters.

The warm electronic thermal enclosure provides thermal interfaces for DPU and ICU electronic boxes between $253 \mathrm{~K}$ and $303 \mathrm{~K}$. The interface plate is an aluminum plate thermally regulated by a heat transfer fluid circulation. The fluid temperature is adjusted by a thermostated bath (Bain Lauda) outside the chamber. To avoid molecular contamination from NI-DPU and NI-ICU, a cold trap will be implemented in front of an aperture in the cover of the enclosure. 


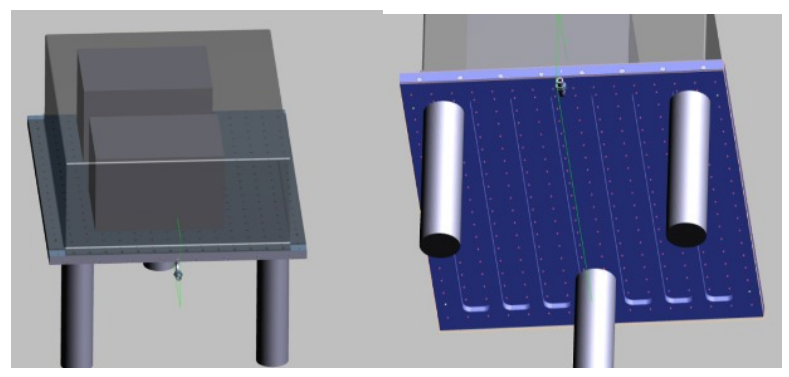

Figure 10: Warm Electronic thermal enclosure

\subsection{Metrology equipment}

In addition to the classical ground support equipment, it is needed to develop a Metrology Verification System (MVS). Its goal is to provide at operational temperature the measurement of references frames set on the telescope simulator and NISP, the knowledge of the coordinates of the object point source provided by the telescope simulator and the measurement of the angle between the telescope simulator optical axis and NISP optical axis. The MVS concept is based on the use of two devices put outside ERIOS chamber:

- A laser tracker, which is a portable coordinate measuring machine provided by Leica that allows extreme accuracy over large distances. The measurement with the laser tracker will be done through a window seeing the reflectors into ERIOS. The current configuration foreseen is to use a curved window. More details on the laser tracker configuration can be found in [4];

- A theodolite, which is a portable angle measuring system, to measure accurate angle between two mirrors, one put on NISP and one on the NI-TS.

The principle of the measurement of the laser tracker is the measurement of the position of reflectors set respectively on the Telescope Simulator (NI-TS) and on NISP instrument. The goal of this measurement is to deliver the position of NISP reference frame $R_{u}$ with respect to NISP object plane $R_{\text {nisp }}$ at Operational Temperature (OT) (i.e. 130K) with accuracy within $+/-150 \mu \mathrm{m}$ to Airbus, the responsible of EUCLID PayLoad Module (PLM), which will align NISP on the EUCLID payload thanks to this information. To provide this information, a sequence of measurement is needed at NISP instrument level:

- Measurement at room temperature with a Coordinate Measurement Machine (CMM) of the frame $R_{u}$ with respect to the laser tracker reference frame $R_{l t}$. $R_{u}$ is a reference frame set at NISP interface with the PLM i.e. NISP feet. $R_{l t}$ is defined by a set of laser tracker reflectors installed on NISP entrance panel P1.

- Measurement at OT with the MVS of $\mathrm{R}_{\mathrm{lt}}$ with respect to $\mathrm{R}_{\text {nisp. }}$. The main difficulty is that $\mathrm{R}_{\text {nisp }}$ cannot be measured directly as it represents the NISP object plane. To be able to provide this information, the TS and the VFP are used. The focus point of the TS will then provide the knowledge of $R_{\text {nisp }}$ through the measurement of a reference frame $R_{t s}$ made of a set of laser tracker reflectors installed on the TS. The knowledge of the transfer matrix between Rts and Rnisp is obtained thanks to a measurement of the TS best focus on the VFP at OT in ERIOS using the MVS. Then during NISP $\mathrm{TB} / \mathrm{TV}$, the transfer matrix between $\mathrm{R}_{\mathrm{ts}}$ and $\mathrm{R}_{\mathrm{lt}}$ is measured with the MVS and allows to obtain the relationship between $R_{l t}$, thus $R_{u}$, and $R_{n i s p}$ that is delivered to Airbus. Figure 11 represents a scheme of the measurement philosophy with the MVS. 


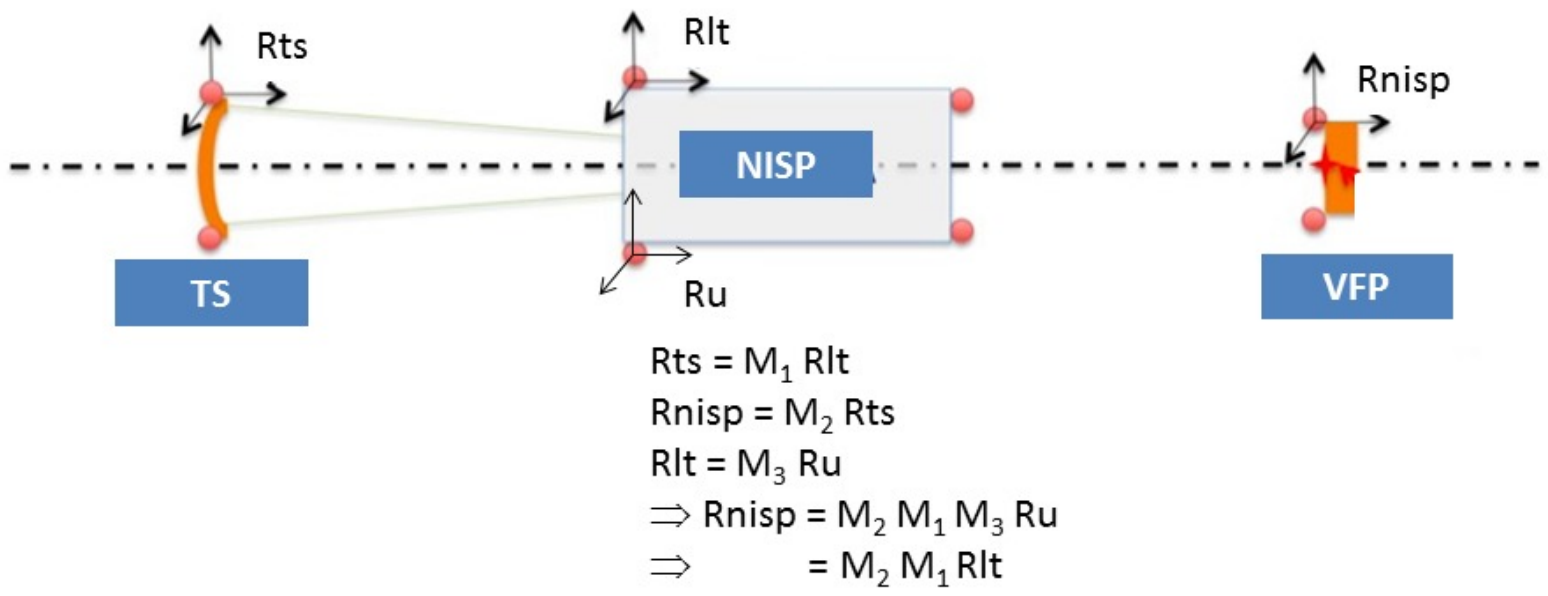

Figure 11. Schematic of the measurement to be done with the MVS. The frames to be measured are shown

The concept and the feasibility study of the MVS are fully described in [4]. We only recall here its mains characteristics. The laser tracker and the theodolite will be placed out of ERIOS chamber and will do the measurement through a window. One has to take into account the measurement errors introduced by the glass in the laser tracker measurement. A mechanical interface will allow the positioning of the both components on ERIOS chamber door to measured reflectors set inside ERIOS. Figure 12 provides a view of the MVS for the NISP performance test. The beam sights are shown to validate the position of the test set-up (NISP and TS) and the laser tracker into ERIOS.
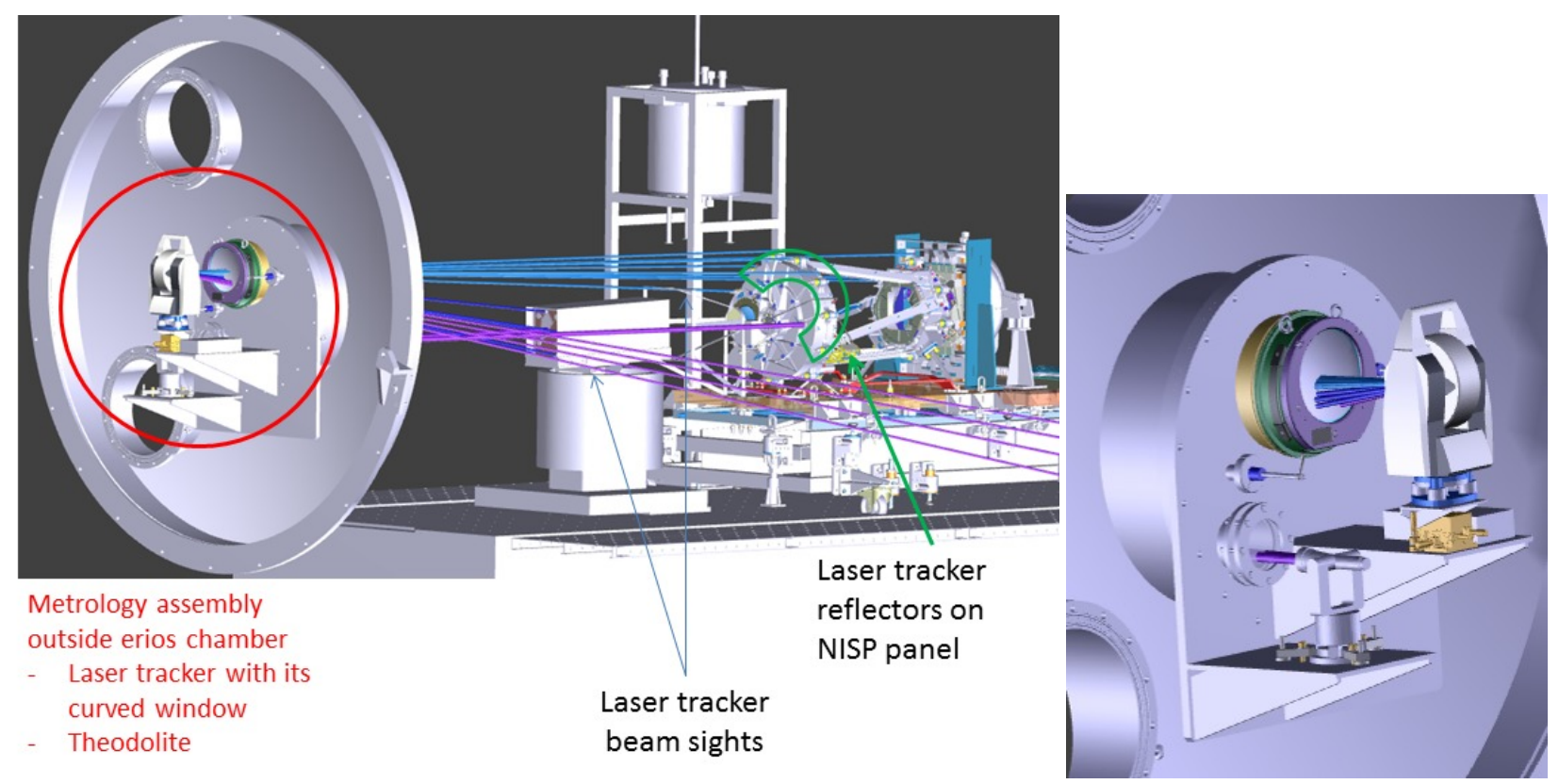

Figure 12. Zoom on the metrology system. Outside erios the laser tracker and the theodolite, inside ERIOS, the reflectors and mirrors.

For the measurement of the angle between NISP and the TS, we use a theodolite put outside ERIOS chamber. A mirror installed is on NISP and a mirror set on the NI-TS. The goal of this measurement is to measure with accuracy the angle of the FoV pointed by the NI-TS on NISP at operational temperature. The MVS parts for the angle measurement are made of: 
- A theodolite installed outside ERIOS chamber. The theodolite will be kept at the same position for all measurements. An adjustment of its position will be done at the beginning of the test,

- A mirror installed on NISP. This mirror will be tilted with respect to P1 plane in order to be seen from the position of the theodolite,

- A set of mirrors will be installed on the back side of the NI-TS. These mirrors will be placed in order to be seen when the NI-TS is set in different positions of the FoV: one for the central position, one for all the four extreme positions of the FoV that have to be measured. The mirrors of the extreme positions are tilted with the FoV angle in order to be seen by the theodolite when the NI-TS is in position.

Figure 13 presents the configuration foreseen for the NISP TB/TV test. The theodolite measurement will be done during the focus test when the NI-TS will be move to point a point in the field of NISP. It will allow to have a good knowledge of the angular position of the NI-VTS with respect to NISP and thus to know precisely what is the FoV angle. This information will be used to reconstruct the "focus" plane of NISP.
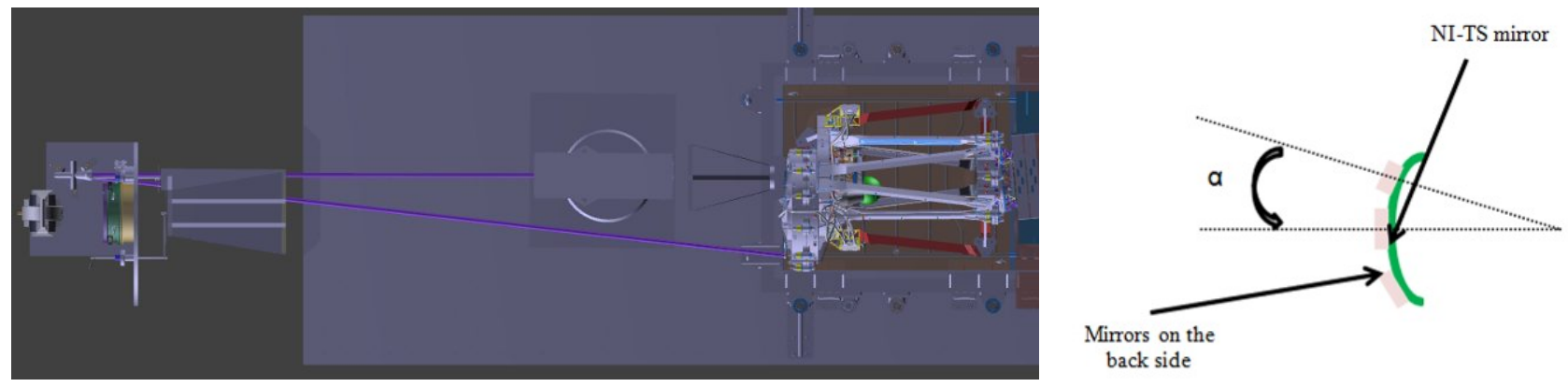

Figure 13. View of the theodolite line of sight inside ERIOS. Right: Scheme of the mirrors on the back side of the NI-TS

The measurement principle for the theodolite is the following:

- The NI-TS is aligned on NISP for the measurement on axis. It is aligned on a chosen pixel of NISP detector,

- Measurement of the angle $\beta$ between NI-TS and NISP is done thanks to the "on-axis" mirror and the mirror on NISP,

- The NI-TS is moved on an off-axis position $\alpha$ : this position corresponds to a position of the NI-TS where a mirror is set on the back side of the telescope, as illustrated on Figure 14,

- The angle between NISP and the NI-TS is measured with the theodolite: $\beta{ }^{\prime}=\beta+\varepsilon$ where $\varepsilon$ is the error due to the translation and rotation stages of the NI-TS. The value of $\beta$ ' is measured with accuracy with the theodolite in order to have an accurate measurement of the position of the NI-TS $\alpha^{\prime}=\alpha-\varepsilon$ giving the $\alpha^{\prime}$ position of the FoV on NISP.
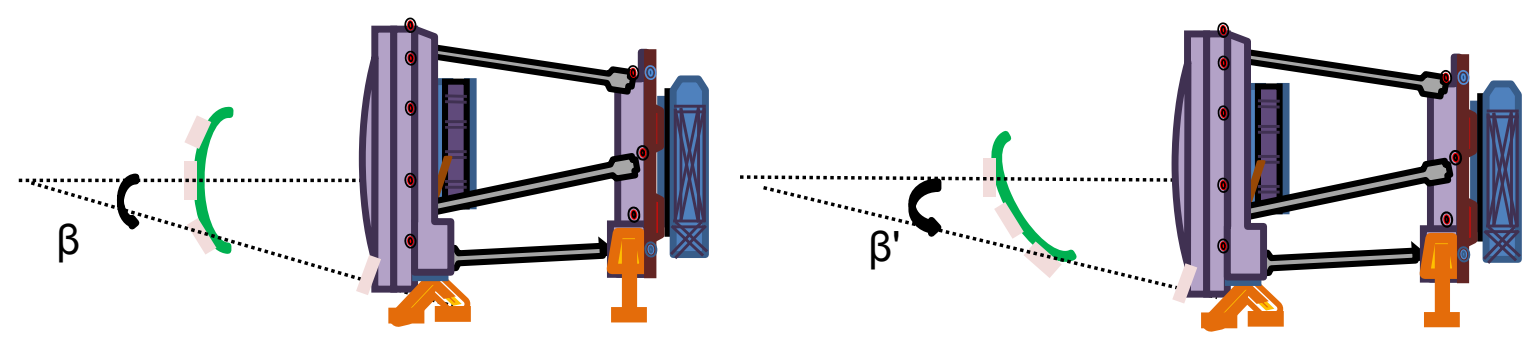

Figure 14. Principle of the theodolite measurement 


\subsection{Control / Command configuration}

The whole VGS should be in interface with NISP and its Electrical GSE (EGSE) for NISP FM TB/TV test. A full description of the EGSE is provided in [5] and [6]. We only present in this article the architecture provided for the test that will allow having a simple interface between all the VGS parts and NISP. The NISP EGSE is made of the NI-WE SCOE, the NISP Central Checkout System (NI-CCS), the EGSE harnesses between the computers and the hardware and the NISP Instrument WorkStation (NI-IWS). All these parts are on an internal network that should be compatible with the PLM network and the NISP performance test. The EGSE is developed for the test of NISP instrument on ground and its goal is to control NISP instrument during the test phase. It will launch the scripts for the test, acquire the data from the detectors and provide all the telemetry from the instrument. For the NISP performance test, it is needed to add an interface between NISP instrument and the NI-TS to control the light sources and the motor of the telescope during the acquisition sequence planned for NISP test. This link is done by the EGSE though the EDEN protocol. In addition, a Database Builder (DB) is foreseen to recover all information from ERIOS, the VGS and NISP EGSE. It will build the high-level database permitting to associate the complete set-up configuration for the instrument and the GSEs to each instrument exposure. It will be a memory of the tests performed at LAM with the VGS. Finally, an analysis computer will be linked to the whole architecture to allow analysis in real time or from remote of the data.

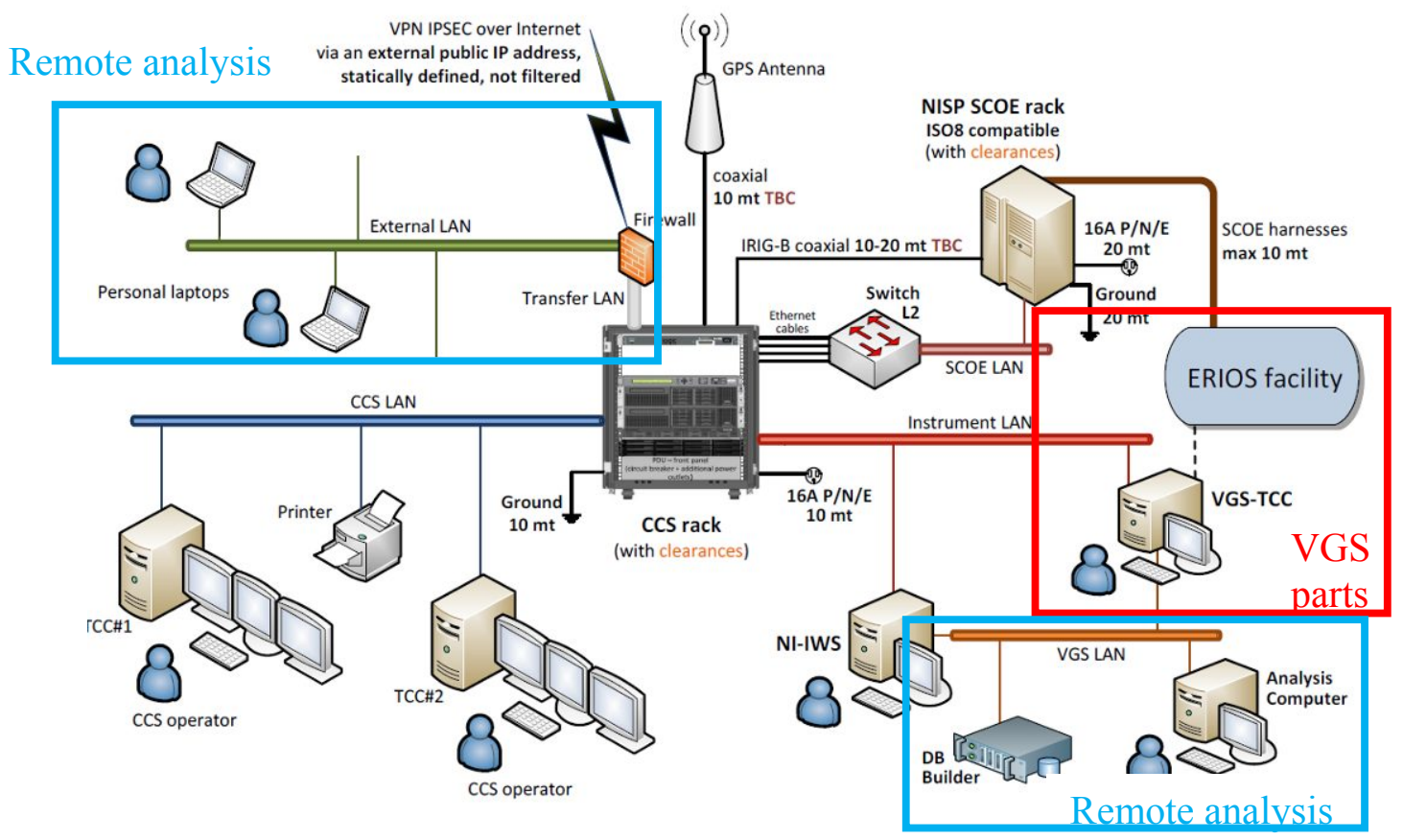

Figure 15. Layout of the control/command system for the NISPperformance test. The parts that are not in square are the parts from NISP EGSE

\section{VGS DEVELOPMENT PLAN}

The development of the VGS will be quite long due to the complexity of this GSE. The goal is that the VGS will be fully operational for Fall 2017 for NISP FM first TB/TV test scheduled for December 2017. To meet this date a development in different phases is foreseen:

- The MVS will be first developed and tested at LAM before March 2017. A test in ERIOS in vacuum will be done using a simulator of NISP and TS reference frames. This will allow the characterization of the metrology, in particular the laser tracker, in vacuum and to define the accuracy that can be reached. An acceptance of the metrology system will be done;

- Final design phase of the TMVS will be done starting in September to freeze the design and need for the thermal parts. The goal is to perform a validation test of the thermal environment for NISP FM in May 2017 when ERIOS will be available. The goal of the acceptance of the TMVS will be to demonstrate the validation of 
the interfaces between the thermal environment and NISP, the stability produced by the interfaces, to validate the temperature of the warm electronics environment and to perform functional tests to ensure good behavior of the system;

- In parallel, the NI-TS will be developed by the Danish consortium. The long lead item is the telescope itself that will arrive for the summer 2017. The other parts of the assembly (the ATS, the VFP, the sources, etc) will be tested before with a telescope test simulator (a kind of "low cost" telescope with no stability and simpler optical geometry). All the parts will be delivered to LAM in early August to perform a full configuration test in ERIOS in cold and vacuum by October 2017. The goal of this full configuration test is important and large. It will validate the measurement the measurement in cold with a laser tracker, we will verify the stability of the focal length of the telescope in cold and verify the movement of the NI-TS assembly in cold (motor response, movement accuracy, repeatability, etc). A first validation of the test procedure will be done in addition to functional tests of the telescope. Finally the thermal behavior of the NI-TS will be demonstrated in ERIOS chamber.

\section{CONCLUSION}

We have presented in this article a complete description of the VGS, a ground support equipment developed at LAM to test NISP instrument at vacuum and cold. The VGS will be used during the performance test campaign of NISP to validate the main functionality of the instrument and its main performance in photometric and spectroscopic modes. The VGS is made of an optical part, which simulates the EUCLID telescope, a set of thermal and mechanical parts to ensure the thermal stability of NISP during the test and of a control / command system to control the instrument together with its GSE during the test. The design of the VGS is done and the manufacturing and test of the VGS parts will be started by September to ensure a delivery of the VGS in one year. The development of the VGS represents a great challenge as many functionalities are required and many institutes are involved.

\section{REFERENCES}

[1] R. Laureijs; G. Racca; L. Stagnaro; J.-C. Salvignol; J. Lorenzo Alvarez, et al, "Euclid mission status," Proc. SPIE 9143, Space Telescopes and Instrumentation 2014: Optical, Infrared, and Millimeter Wave, 91430H (2014)

[2] T. Maciaszek; A. Ealet; K. Jahnke; E. Prieto; R. Barbier, et al., "Euclid near infrared spectrophotometer instrument concept and first test results at the end of phase B," Proc. SPIE 9143, Space Telescopes and Instrumentation 2014: Optical, Infrared, and Millimeter Wave, 91430K (2014)

[3] M. Cropper; S. Pottinger; S.-M. Niemi; J. Denniston; R. Cole, et al., "VIS: the visible imager for Euclid," Proc. SPIE 9143, Space Telescopes and Instrumentation 2014: Optical, Infrared, and Millimeter Wave, 91430J (2014)

[4] A. Costille; F. Beaumont; E. Prieto; M. Carle, C. Fabron; "Three dimensional metrology inside a vacuum chamber," Proc. SPIE 9912, Space Telescopes and Instrumentation 2016: Optical, Infrared, and Millimeter Wave, 9912-154 (2016)

[5] E. Franceschi; et al., "EGSE customization for the Euclid NISP Instrument AIV/AIT activities," Proc. SPIE 9904, Space Telescopes and Instrumentation 2016: Optical, Infrared, and Millimeter Wave, 9904-97 (2016)

[6] M. Trifoglio; et al., "Instrument Workstation for the EGSE of the Near Infrared Spectro-Photometer instrument (NISP) of the EUCLID mission," Proc. SPIE 9904, Space Telescopes and Instrumentation 2016: Optical, Infrared, and Millimeter Wave, 9904-231 (2016) 